\title{
CARACTERIZAÇÃO SOCIODEMOGRÁFICA E SOBRECARGA DOS CUIDADORES INFORMAIS DE IDOSOS APÓS ACIDENTE VASCULAR CEREBRAL
}

\author{
Claudia Maria Ferrony Rivas'; Eduardo Marques Machado ${ }^{2}$; Emilly Barcelos \\ Petter ${ }^{3}$; Natalia Cassol Bolzan ${ }^{4}$; Rafaela Cantarelli5; Clandio Timm Marques ${ }^{6}$; \\ Maria Helena Gehlen ${ }^{7}$; Naiana Oliveira dos Santos ${ }^{8}$.
}

\section{RESUMO}

A pesquisa tem o objetivo de descrever as características sociodemográficas e identificar o grau de sobrecarga dos cuidadores informais de idosos após acidente vascular cerebral (AVC) acompanhados pelo programa de Atenção Domiciliar (AD). Estudo transversal, descritivo, realizado em uma Estratégia Saúde da Família (ESF) localizada no município de Santa Maria, Rio Grande do Sul. Para a caracterização sociodemográfica dos cuidadores, utilizou-se um instrumento feito pelos pesquisadores, para avaliação da sobrecarga dos cuidadores informais aplicou-se a Escala de Zarit. A maioria dos cuidadores informais eram mulheres, idade média de 48,5 anos, 38,4\% tinham mais de 60 anos, baixa escolaridade, eram pertencentes ao círculo familiar dos idosos, casadas ou em união estável, com modesta renda familiar. Mais da metade da amostra sentia-se sobrecarregada. Considera-se que o conhecimento destacado pode auxiliar na construção de um plano de cuidados com maior qualidade para usuários idosos após AVC em AD e para seus cuidadores.

Palavras-chave: Enfermagem; Atenção Domiciliar; Pesquisa.

Eixo Temático: Atenção Integral e Promoção à Saúde (AIPS).

\section{INTRODUÇÃO}

O acidente vascular cerebral (AVC) é o surgimento de um déficit neurológico que ocorre subitamente ocasionado por complicações nos vasos do cérebro. Caracteriza-se o AVC em dois tipos, o AVC isquêmico e o hemorrágico. No Brasil, o

\footnotetext{
${ }^{1}$ Acadêmica de Enfermagem-Universidade Franciscana-UFN e-mail: claudiamfrivas@gmail.com

${ }^{2}$ Acadêmico de Enfermagem-Universidade Franciscana-UFN e-mail: eduardomachado051@gmail.com

${ }^{3}$ Acadêmica de Enfermagem-Universidade Franciscana-UFN e-mail: emillypetter@gmail.com

${ }^{4}$ Acadêmica de Enfermagem-Universidade Franciscana-UFN e-mail: natalia.bolzan23@gmail.com

${ }^{5}$ Acadêmica de Enfermagem-Universidade Franciscana-UFN e-mail: ra.cantarelli0909@gmail.com

${ }_{6}^{6}$ Professor do curso de Matemática-Universidade Franciscana e-mail: clandio@ufn.edu.br

${ }^{7}$ Professora do curso de Enfermagem-Universidade Franciscana-UFN e-mail: gehlenmh@gmail.com

8 Orientadora e Pesquisadora responsável pela pesquisa. Professora do curso de Enfermagem-

Universidade Franciscana-UFN e-mail: naiaoliveira07@gmail.com
} 
AVC representa a segunda causa de mortes na população e no mundo a doença é responsável pela principal causa de incapacidades (SBDCV, 2021).

Portanto, quando o AVC não causa o óbito, acaba por trazer incapacidades cognitivas e funcionais no indivíduo acometido pela síndrome neurológica, ocasionando a dependência de cuidados após a saída do ambiente hospitalar (BENJAMIN et al., 2017).

O aumento das doenças crônicas tais como hipertensão arterial sistêmica, diabetes mellitus e cardiopatias na população idosa combinado com a predisposição genética, modificações inerentes ao processo de envelhecimento e a fatores de risco como uso de tabaco, vida sedentária, má alimentação e obesidade faz com o AVC ocorra com maior frequência em idosos (SANTOS e WATERS, 2020).

Tendo em vista as particularidades de se realizar o cuidado dos idosos após AVC no ambiente domiciliar, destaca-se no Sistema único de Saúde (SUS) o programa de Atenção Domiciliar (AD), uma modalidade de atenção à saúde que engloba ações de promoção da saúde, prevenção, tratamento, reabilitação e paliação em domicílio, articulada com as Redes de Atenção à Saúde (RAS) (BRASIL, 2016).

A AD considerando a complexidade e especificidades dos usuários e os contextos nos quais os usuários estão inseridos, torna-se um importante modelo complementar com capacidade para produzir o cuidado e realizar o processo de trabalho em saúde com eficiência (PROCÓPIO et al., 2019).

No cuidado realizado no domicílio destaca-se a importância da figura do cuidador informal, indivíduo com laços consanguíneos ou vínculo afetivo com o usuário dependente de cuidados (BRASIL, 2013). No que se concerne aos sujeitos após AVC, observa-se o impacto temporário ou definitivo nas atividades de vida diária, desta forma torna-se primordial no contexto de vida desta pessoa a presença do cuidador (TAVARES et al., 2020).

Diferentes atividades cotidianas estão relacionadas ao ato de cuidar, percebese que essa rotina de afazeres pode resultar na sobrecarga do cuidador, podendo afetar na sua saúde, como também prejudicar a qualidade do cuidado prestado (BOM, SÁ e CARDOSO, 2017). Identificar o nível de sobrecarga e as características 
dos cuidadores informais, possibilita facilitar a elaboração do planejamento dos cuidados realizados no domicílio pela equipe multiprofissional de saúde, proporcionando uma atenção à saúde mais direcionada (SILVA et al., 2016).

Dentro deste contexto a presente pesquisa tem o objetivo de descrever as características sociodemográficas e identificar o grau de sobrecarga dos cuidadores de idosos após AVC acompanhados pelo programa de AD.

\section{METODOLOGIA}

Trata- se de um estudo transversal, descritivo. O campo de pesquisa foi uma Estratégia Saúde da Família (ESF) localizada no município de Santa Maria, Rio Grande do Sul. Optou-se pela referida ESF por conveniência, em virtude de o grande número de usuários em AD.

Foram sujeitos da pesquisa os cuidadores informais de usuários idosos após AVC assistidos pelo programa de AD. Assim, de 53 usuários/cuidadores cadastrados em AD da ESF, incluíram-se 13 cuidadores que preenchiam os critérios de inclusão sendo eles: cuidadores não remunerados de idosos após AVC e dependentes de cuidados para uma ou mais atividades de vida diária; possuindo ou não laços consanguíneos com o usuário idoso; com idade mínima de 18 anos e com tempo de cuidado no domicílio pós alta hospitalar do idoso após AVC de no mínimo 15 dias. Usuários não contactados após três tentativas em dias e turnos diferentes foram excluídos do estudo.

A coleta de dados ocorreu de julho a setembro do ano de 2020, através de entrevistas feitas no domicílio dos idosos, agendadas previamente pelos agentes comunitários de saúde responsáveis pelo cadastramento dos indivíduos do programa de AD.

A visita para a entrevista foi realizada em ambiente aberto na residência do cuidador, teve duração média de 30 minutos e respeitou as recomendações de prevenção da pandemia do vírus COVID-19. Sendo assim, foram utilizados equipamentos de proteção individual pelos pesquisadores e manteve-se o distanciamento necessário. 
Para a caracterização sociodemográfica dos cuidadores, utilizou-se um instrumento feito pelos pesquisadores com propósito de se obter as seguintes informações: idade, sexo, escolaridade, estado conjugal. Outros dados também foram coletados como, grau de parentesco do cuidador com o idoso, renda familiar, se o cuidador recebia ajuda de outra pessoa para o cuidado e se o cuidador se sentia sobrecarregado.

Para avaliação da sobrecarga dos cuidadores informais que auto referiram sobrecarga no primeiro questionário aplicou-se a Escala de Zarit (ZARIT et al., 1980). A avaliação foi realizada sem a presença do idoso. Esta escala teve sua tradução e validação para o Brasil em 2002, possui 22 questões de múltipla escolha, com as respostas variando de 0 a 88 pontos, quanto maior a pontuação, maior é a sobrecarga, sendo assim 61 a 88 pontos, correspondem à sobrecarga severa; 41 a 60 pontos: sobrecarga moderada a severa; 21 a 40 pontos, sobrecarga leve a moderada e menos de 21 pontos ausência de sobrecarga ou sobrecarga mínima (SEQUEIRA, 2010).

A análise dos dados foi feita pelo programa software Statistical Package for the Social Sciences (SPSS) versão 21.0. As variáveis quantitativas foram expressas como média e desvio padrão ou mediana e intervalo interquartil, de acordo com a normalidade. As variáveis categóricas foram expressas como frequências absolutas ou relativas.

Em consonância com a Resolução 466/12 do Conselho Nacional de Saúde a pesquisa foi realizada após aprovada por um Comitê de Ética em Pesquisa, sob o Parecer no 3.308.174 em 07/05/2019. Os aspectos éticos foram respeitados e a produção de dados foi feita após a assinatura do Termo de Consentimento Livre e Esclarecido. A presente pesquisa é integrante de um estudo maior intitulado "Avaliação Multidimensional de Usuários e Cuidadores do Programa de Atenção Domiciliar".

\section{RESULTADOS E DISCUSSÕES}

Entre as características sociodemográficas dos cuidadores informais de idosos após AVC em AD destaca-se que a maioria eram mulheres $(76,92 \%)$ com 
média de idade de 48,5 anos, 38,4\% cuidadores possuíam mais de 60 anos e na grande maioria tinham laços consanguíneos com os idosos acometidos por AVC (70,3\%). Quanto ao estado civil, $61,5 \%$ eram casadas ou moravam com companheiro.

Ao comparar o resultado com outros estudos, observa-se o predomínio de mulheres exercendo 0 ato de cuidar (AIRES et al., 2017; LIMA et al., 2020; CONCEIÇÃO et al., 2021). Esse achado demonstra uma perspectiva histórica vigente ainda em nossa sociedade, que vincula o cuidado dos familiares a uma responsabilidade inerente ao sexo feminino (FERREIRA, ISAAC e XIMENES, 2018).

No que se refere a faixa etária, um estudo semelhante (SANTOS-ORLANDI et al., 2017). corrobora com os resultados da pesquisa, apresentando uma média de idade entre os cuidadores de 45,5 anos, como também evidência na amostra cuidadores informais com idade maior que 60 anos. $O$ mesmo estudo evidencia que se faz necessário planejar uma sistematização de atenção de modo individual direcionado ao perfil de cuidadores idosos, enfatizando a promoção da saúde e à prevenção de agravos no cenário da ESF.

No que tange ao grau de parentesco a presente pesquisa mostra que $70,3 \%$ cuidadores informais de idosos após AVC em AD tinham laços consanguíneos com os idosos. Esses resultados estão em consonância com outros estudos que trazem como principal cuidador informal uma pessoa do círculo familiar da pessoa dependente de cuidados (NEVES et al., 2019; LOPES et al., 2020; SOUZA, FARINHA e TRETTENE, 2019; MAMANI et al., 2019).

Fazer parte da família comumente influencia na atribuição de ser cuidador, já que ao ter o vínculo de afeto, os familiares acabam por sentir obrigatoriedade moral para se encarregar do cuidar (FERREIRA, ISAAC e XIMENES, 2018).

Quanto ao estado civil outros estudos chegaram a resultados semelhante aos dessa pesquisa, sendo os cuidadores informais majoritariamente casados ou mantendo relacionamento estável (CARO, COSTA e CRUZ, 2018; MAMANI et al., 2019).

Em relação a escolaridade prevaleceu a baixa escolaridade demonstrando que $53,84 \%$ possuíam o ensino fundamental completo ou incompleto. Outras 
pesquisas evidenciam a baixa escolaridade, mostrando a prevalência de cuidadores com o ensino fundamental incompleto (ALMEIDA et al., 2018; SANTOS-ORLANDI et al., 2019; JESUS, ORLANDI e ZAZETTA, 2018; CARO, COSTA e CRUZ, 2018; CONCEIÇÃO et al., 2021). Infere-se que o grau de instrução pode afetar na capacidade de aquisição de conhecimento para realizar o cuidado, pois a capacidade de aprender está vinculada a uma eficiente compreensão das ações de saúde a serem desempenhadas pelo cuidador (ALMEIDA et al., 2018).

No que concerne a ocupação dos cuidadores 53,84\% estavam desempregados no momento da entrevista e quanto a renda familiar a média observada foi de 1,5 salários-mínimos $( \pm 1,050)$ (baseado no salário-mínimo brasileiro vigente no ano de 2020: $R \$ 1045,00)$. Uma revisão integrativa sobre o perfil dos cuidadores de usuários pós AVC, mostra que majoritariamente os cuidadores estão desempregados e possuem baixa renda financeira (LIMA et al., 2019). Outros estudos (BIERHALS et al., 2020; AIRES et al., 2017; CARO, COSTA e CRUZ, 2018) também ratificam esses dados. Jesus, Orlandi e Zazetta (2019) igualmente pontuam sobre a modesta renda familiar entre os cuidadores de usuários em $A D$ e postulam que em muitos casos, o estar desempregado, ocorre pelo fato de o cuidador ter que sair de sua ocupação laboral em decorrência da tarefa de cuidar de seu familiar.

Da amostra, 53,8\% dos cuidadores informais referiram que recebem auxílio para prestar o cuidado ao idoso. Muitas vezes a participação do cuidado ocorre por outros familiares do idoso (JESUS, ORLANDI E ZAZETTA, 2019). Situação que aparentemente pode amenizar a carga de trabalho do cuidador, como também trazer maior sobrecarga a rotina familiar.

Entre os cuidadores informais 58,33\% relataram sobrecarga relacionada ao ato de cuidar, 41,66\% apresentaram sobrecarga moderada e 16,66\%, sobrecarga de moderada a severa.

Estudo sobre o perfil e sobrecarga dos cuidadores informais de idosos dependentes expõe que 90,4\% indicaram algum nível de sobrecarga por meio da aplicação da escala de sobrecarga de Zarit (CONCEIÇÃO et al., 2021). Outra pesquisa que utilizou da mesma escala para avaliar a sobrecarga dos cuidadores 
EDUCAÇÃO, SAÚDE

ETECNOLOGIA

26 A 28 DE OUTUBRO DE 2021

\section{QuFN}

informais mostra que $83,5 \%$ dos cuidadores apresentaram sobrecarga grave associado aos cuidadores que não exerciam atividade laboral (BRANDÃO et al., 2017).

Muitos cuidadores demonstram-se sobrecarregados o que pode impactar em sua qualidade de vida e nos cuidados dispensados ao idoso (SOUZA, FARINHA e TRETTENE, 2019), indicando uma repercussão negativa, tanto no aspecto físico, psicológico, emocional e financeiro (TRINDADE et al., 2017). De modo geral, o cuidador não está pronto para se responsabilizar para dar o apoio e o suporte que a pessoa a ser cuidada, necessita e precisa receber orientações em relação as atividades que deverá assumir (JESUS, ORLANDI e ZAZETTA, 2019). Dessa maneira, é essencial o planejamento e intervenções dos profissionais da saúde com intuito de tentar reduzir a sobrecarga, promover a qualidade de vida e consequentemente qualificar o cuidado fornecido ao usuário dependente (SOUZA, FARINHA E TRETTENE, 2019).

Assim como é importante que os profissionais da saúde avaliem a sobrecarga dos cuidadores, orientem os cuidadores para que conciliem o cuidado com outras tarefas cotidianas e identifiquem serviços de suporte comunitário (CARO, COSTA e CRUZ, 2018). Portanto, é incumbência da equipe multiprofissional da $A D$ e prioritariamente dos profissionais enfermeiros, responsáveis diretos pelo cuidado, pautar as intervenções e ações em saúde focadas em todos envolvidos no processo de cuidar dos idosos após AVC, considerando o contexto familiar, comunitário e social, suas características pessoais e suas singularidades.

\section{CONCLUSÃO}

Esta pesquisa proporcionou revelar a caracterização sociodemográfica dos cuidadores de idosos após AVC vinculados ao programa de AD de uma ESF do município de Santa Maria/RS. Os resultados demonstraram que a maioria eram mulheres, com média de idade de 48,5 anos, 38,4\% tinham mais de 60 anos, baixa escolaridade, eram pertencentes ao círculo familiar dos idosos, casadas ou em união estável, desempregadas, com modesta renda familiar, e grande parcela dos cuidadores recebiam auxílio para realizar o cuidado. Constatou-se também que mais da metade da amostra sentia-se sobrecarregado, apresentando sobrecarga de 


\section{QUFN}

moderada a moderada e severa.

A respeito das limitações desse estudo, pode-se citar o tamanho pequeno da amostra e o fato de ela ser feita por conveniência. A inclusão de outras EFS do município, obteria dados mais fidedignos sobre o perfil dos cuidadores informais de idosos pós AVC e sobre o nível de sobrecarga desses indivíduos.

Apesar das limitações da pesquisa considera-se que o conhecimento destacado sobre o perfil dos cuidadores informais pode sensibilizar os profissionais da saúde para atuar considerando a perspectiva do cuidador e auxiliar na construção de um plano de cuidados mais eficiente e com maior qualidade para os usuários idosos pós AVC em AD e para seus cuidadores.

\section{AGRADECIMENTOS}

A Universidade Franciscana-UFN e a Fundação de Amparo à Pesquisa do Estado do Rio Grande do Sul-FAPERGS.

\section{REFERÊNCIAS}

AIRES, M. et al. Associação entre responsabilidade filial no cuidado aos pais e sobrecarga dos cuidadores. Rev Bras Enferm. v. 70, n.4, 2017. Disponível em: <https://doi.org/10.1590/0034-7167-2017-0133> Acesso em: 10 set 2021.

ALMEIDA, L. de P. B et al. Características sociais e demográficas de idosos cuidadores e motivos para cuidar da pessoa idosa em domicílio. REME • Rev Min Enferm $\quad$ v. 22: e-1074. Disponível em: <https://cdn.publisher.gn1.link/reme.org.br/pdf/e1074.pdf $>$ Acesso em:12 set 2021.

BENJAMIN, E. J. et al. Heart disease and stroke statistics-2017 update: a report from the American Heart Association. American Heart Association Statistics Committee and Stroke Statistics Subcommittee. Ahajournals, v.135, n.10, p.146-603, 2017. 
Disponível

em:<

https://www.ahajournals.org/doi/epub/10.1161/CIR.0000000000000485> Acesso em: 09 set 2021.

BIERHALS, C.C.B.K., et al. Use of health services by elderly people post-stroke: A randomized controlled trial. Rev Gauch Enferm v. 41(esp), 2020. Disponível em: <https://doi.org/10.1590/1983-1447.2020.20190138> Acesso em: 10 set 2021.

BOM F.S., SÁ, S.P.C, CARDOSO, R.D.S.S. Sobrecarga em cuidadores de idosos. J Nurs UFPE online, p.11, n.1, p.160-4. Disponível em < https://doi.org/10.1590/198122562018021.170155> Acesso em: 11 set 2021

BRANDÃO, F. S. R. et al. Sobrecarga dos cuidadores de idosos assistidos por um serviço de atenção domiciliar. Revista de Enfermagem UFPE on line, [S.I.], v. 11, n. 1, p. 272-279, dez. 2016. Disponível em: $<$ https://periodicos.ufpe.br/revistas/revistaenfermagem/article/view/11905>. Acesso em: 16 set. 2021.

BRASIL. Ministério da Saúde. Portaria no 825, de 25 de abril de 2016. Redefine a Atenção Domiciliar no âmbito do Sistema Único de Saúde (SUS) e atualiza as equipes habilitadas [internet]. Brasília, DF: Ministério da saúde; 2016. Disponível em: <http://bvsms.saude.gov.br/bvs/saudelegis/gm/2016/prt0825 2504 2016.html> Acesso em: 09 set 2021.

BRASIL. Ministério da Saúde. Secretaria de Atenção à Saúde. Diretrizes de atenção à reabilitação da pessoa com acidente vascular cerebral, Brasília/DF, 2013.

Disponível

em:< https://bvsms.saude.gov.br/bvs/publicacoes/diretrizes atencao reabilitacao acident e vascular cerebral.pdf>. Acesso em 09 set 2021. 
CARO, C. C., COSTA, J. D., CRUZ, D. Burden and Quality of Life of Family Caregivers of Stroke Patients. Occupational therapy in health care, v. 32, n. 2, p. 154-171, 2018. Disponível em:<https://doi.org/10.1080/07380577.2018.1449046> Acesso em: 13 set 2021.

CONCEIÇÃO H.N. da et al. Perfil e sobrecarga dos cuidadores informais de idosos dependentes. Research, Society and Development, v. 10, n. 6, e47210616061, 2021. Disponível em:<http://dx.doi.org/10.33448/rsd-v10i6.16061> Acesso em: 13 set 2021.

FERREIRA, C. R.; ISAAC, L.; XIMENES, V. S. Cuidar de idosos: um assunto de mulher? Est. Inter. Psicol., Londrina, v. 9, n. 1, p. 108-125, jun. 2018. Disponível em:<http://pepsic.bvsalud.org/scielo.php?script=sci arttext\&pid=S2236-

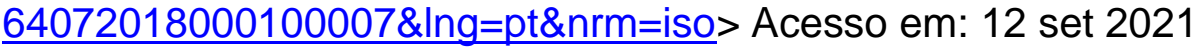

JESUS, I.T. M. de; ORLANDI, A. A. dos S. e ZAZETTA, M. S. Burden, profile and care: caregivers of socially vulnerable elderly persons. Revista Brasileira de Geriatria e Gerontologia [online]. v. 21, n. 02, p.194-204, 2018. Disponível em:< https://doi.org/10.1590/1981-22562018021.170155> Acesso: 12 set 2021

LIMA, K.P. et al. Acidente vascular cerebral: um olhar para o perfil do cuidador. Revista Eletrônica Acervo Saúde, v. 12, n. 11, e4677, nov. 2020. Disponível em:< https://acervomais.com.br/index.php/saude/article/view/4677>. Acesso em: 12 set 2021

LOPES, C. C. et al. Associação entre a ocorrência de dor e sobrecarga em cuidadores principais e o nível de independência de idosos nas atividades de vida diária: estudo transversal. Cad. saúde colet., Rio de Janeiro, v. 28, n. 1, p. 98-106, Mar. 2020. Disponível em:< http://dx.doi.org/10.1590/1414-462x202028010184> Acesso em: 12 set 2021. 
MAMANI, A. R. N. et al . Cuidador de idosos: conhecimentos, atitudes e práticas sobre quedas e sua prevenção. Rev. Bras. Enferm., Brasília , v. 72, supl. 2, p. 119126, 2019. Disponível em:< https://doi.org/10.1590/0034-7167-2018-0276>. Acesso em: 13 set 2021

NEVES, A. C. de J. et al. Atenção domiciliar: perfil assistencial de serviço vinculado a um hospital de ensino. Physis: Revista de Saúde Coletiva [online]. v. 29, n. 02, 2019. Disponível em:< https://doi.org/10.1590/S0103-73312019290214 > Acesso em: 13 set 2021.

PROCÓPIO, L. C. R., et al. A Atenção Domiciliar no âmbito do Sistema Único de Saúde: desafios e potencialidades. Saúde debate, Rio de Janeiro, v. 43, n. 121, p. 592-604, 2019. Disponível em:

< https://www.scielo.br/j/sdeb/a/Yz6YQWK9z67wagrssVY7LBk/?lang=pt>

Acesso em: 09 set 2021

SANTOS-ORLANDI, A. A. dos et al. Perfil de idosos que cuidam de outros idosos em contexto de alta vulnerabilidade social. Escola Anna Nery [online]. v. 21, n. 1, 2017. Disponível em:<https://doi.org/10.5935/1414-8145.20170013> Acesso em: 12 set 2021.

SANTOS, L.B. de; WATERS, C. Perfil epidemiológico dos pacientes acometidos por acidente vascular cerebral: revisão integrativa. Braz. J. of Develop., Curitiba, v. 6, n. 1, p. 2749-2775 jan. $2020 . \quad$ Disponível em:<https://www.brazilianjournals.com/index.php/BRJD/article/view/6186>. Acesso em: 09 set 2021.

SEQUEIRA, C.A.C. Adaptação e validação da Escala de Sobrecarga do Cuidador de Zarit. Rev Referência, v.12, n.2, p. 9-16, 2010. Disponível em:< http://www.indexf.com/referencia/2010pdf/12-0916.pdf> Acesso em: 12 set 2021. 
SILVA, J.K. et al. Perfil de cuidadores familiares de idosos após o Acidente Vascular Cerebral. Rev. Enferm. UFPE, v. 10, n. 10, p. 3727-3733, 2016. Disponível em:< https://periodicos.ufpe.br/revistas/revistaenfermagem/article/view/11437>.Acesso em 11 set 2021.

Sociedade Brasileira de Doenças Cerebrovasculares (SBDCV). Acidente vascular cerebral, 2021. Disponível em:<https://avc.org.br/noticias/acidente-vascularcerebral/> Acesso em 09 set 2021.

SOUZA, B.C.C. de; FARINHA, F.T., TRETTENE, A dos S. O cuidar do paciente com Acidente Vascular Cerebral: repercussões no cuidador informal, J Health Sci Inst. v.37, n.3, p. 264-71, 2019. Disponível em: <https://repositorio.unip.br/wpcontent/uploads/2020/12/13V37 n3 2019 p264a271.p $\underline{\mathrm{df}}>$ Acesso em 13 set 2021

TAVARES, M. T. S. et al., Construção e validação de uma tecnologia educativa do tipo cartilha para cuidadores familiares de indivíduos vítimas de avc. Encontro de Extensão, Docência e Iniciação Científica (EEDIC), [S.I.], v. 7, nov, 2020. Disponível em:

$<$ http://publicacoesacademicas.unicatolicaquixada.edu.br/index.php/eedic/article/vie $\underline{w} / 4222>$ Acesso em: 11 set 2021

TRINDADE, I.et al.,Caracterização do grau de sobrecarga dos cuidadores de utentes dependentes da Unidade de Saúde Familiar USF Descobertas. Rev Port Med Geral Fam., v. 33, n. 3, p.178-86, $2017 . \quad$ Disponível em: <https://www.rpmgf.pt/ojs/index.php/rpmgf/article/view/12160> Acesso em 12 set 2021.

ZARIT, S. H. et al. Relatives of the impaired elderly: correlates of feelings of burden. The Gerontologist, v. 20, n.6, p. 649-655, 1980 Disponível em: <https://doi.org/10.1093/geront/20.6.649> Acesso em 10 set 2021. 